\section{Acute myositis associated with concurrent infection of rotavirus and norovirus in a 2-year-old girl}

\author{
Kei Yamamoto,1 Seiji Fukuda, 1 \\ Yuichi Mushimoto, ${ }^{1}$ Noriaki Minami,1 \\ Rie Kanai,1 Kazuki Tsukamoto,² \\ Seiji Yamaguchi1 \\ 1Department of Pediatrics, Shimane \\ University School of Medicine; \\ 2Department of Pediatrics, National \\ Hospital Organization Hamada Medical \\ Center, Japan
}

\begin{abstract}
Rotavirus and norovirus are common pathogens associated with gastroenteritis in children. Although rotavirus occasionally induces central nervous system disease, only 3 cases with rotavirus-induced acute myositis have been reported in the English literature. We recently treated a female patient with acute myositis associated with gastroenteritis induced by concurrent infection with rotavirus and norovirus. Having suffered from gastroenteritis for 3 days, she suddenly developed myositis affecting her lower extremities with concomitant creatine kinase elevation. Herein, we present our patient and review the previous cases including those reported in the Japanese literature.
\end{abstract}

\section{Introduction}

Rotavirus and norovirus are common pathogens associated with gastroenteritis in children. ${ }^{1}$ Although they could induce central nervous system disease, only 3 cases with rotavirus-induced acute myositis have been reported in the English literature. ${ }^{2-4}$ Acute myositis is often caused by various infectious pathogens, such as mycoplasma pneumonia, influenza virus and adenovirus. ${ }^{5}$ In contrast, acute myositis associated with rotavirus or norovirus is seldom reported. Rotavirus-associated muscle damage can be life threatening as a result from rhabdomyolysis or cardiac muscle impairment, 4,6 but the mechanism responsible for the development of muscle damage remains unknown. Therefore, additional case reports are warranted to determine the epidemiology and pathogenesis underlying rotavirus-associated muscle damage.

\section{Case Report}

A 2.5-year-old female patient was referred to our department complaining that she was not able to move her lower extremities coincidence with elevated serum creatine phosphokinase (CK) and CK-MB levels. She had been receiving infusion for fever, vomiting and diarrhea resulting from rotavirus-associated gastroenteritis for 3 days before being referred to our hospital. Diagnosis of rotavirus-induced gastroenteritis was made based on frequent diarrhea and the presence of rotavirus antigen in her stool. In addition to Group A rotavirus, norovirus (G2) was also isolated from her stool in the subsequent analysis. Her fever and diarrhea resolved in the first 3 days. However, on day 4 , she was reluctant to move her lower extremities and was unable to stand or walk (Figure 1), whereas she was able to move her upper extremities normally. While her serum CK level was 133 IU/L (reference: $36-216 \mathrm{IU} / \mathrm{L}$ ) the day before infusion (day 1), it was elevated to 5547 IU/L on day 4 when she was not able to move her legs. CK-MB was $122 \mathrm{IU} / \mathrm{L}$, which was over the normal limit (reference value $<25 \mathrm{IU} / \mathrm{L}$ ). Hence, she was suspected to be suffering from acute myocarditis, one of several rare complications associated with rotavirus infection. ${ }^{5}$ Her past history was unremarkable with no evidence of any developmental delays, although she did have a respiratory tract infection approximately 13 days before hospitalization. She had not received a vaccination against rotavirus. There was no tenderness on her leg muscle. Her patellar tendon reflex and Achilles tendon reflex were normal on both sides. She showed no signs of consciousness disturbance or cerebellar ataxia. Neither abdominal masses nor breathing difficulties were identified. Her cardiac sound and echocardiography (ECG) were normal. Echocardiography indicated her ejection fraction index was $79 \%$ without any signs of cardiac effusion. Chest X-ray revealed that her cardio thoracic ratio was $55 \%$ and pulmonary effusion was not detected. Troponin T was negative, and BNP was $11.2 \mathrm{pg} / \mathrm{mL}$ (reference value $<20$ $\mathrm{pg} / \mathrm{mL}$ ). The titer of serum CK was 4,930 IU/L, but $99.1 \%$ of CK was MM type and only $0.7 \%$ was CK-MB, as determined by electrophoresis of CK fraction. These findings suggested that cardiac muscle damage was highly unlikely, which was opposed to what was initially suspected. Serum aspartate aminotransferase (AST), alanine aminotransferase (ALT) and lactate dehydrogenase (LDH) were $240 \mathrm{IU} / \mathrm{L}, 66 \mathrm{IU} / \mathrm{L}$ and $352 \mathrm{IU} / \mathrm{L}$, respectively. Aldolase was $55.5 \mathrm{IU} / \mathrm{L}$ (reference value $<7.5 \mathrm{IU} / \mathrm{L}$ ). Serum $\mathrm{Na}, \mathrm{K}, \mathrm{Cl}, \mathrm{Mg}$ and $\mathrm{Ca}$ were all in a normal range. Soluble IL-2 receptor was elevated to 2,509 U/ml. Blood TSH, free T3 and free T4 were normal. Mycoplasma antibody was negative. Influenza virus, adenovirus, RS virus and Streptococcus pyogenes were not
Correspondence: Seiji Fukuda, Department of Pediatrics, Shimane University School of Medicine, 89-1 Enya, Izumo, Shimane 693-8501, Japan.

Tel.: +81.853.20.2217 - Fax: +81-853.20.2215.

E-mail: sfukuda@med.shimane-u.ac.jp

Key words: Rotavirus; norovirus; acute myositis; creatine kinase.

Acknowledgements: we thank Mr. John Telloyan for English assistance and the Shimane Prefecture Infectious Disease Surveillance Center for isolating the rotavirus and norovirus.

Contributions: KY and SF participated in the patient's care, interpreted the laboratory data, and wrote the manuscript; YM, NM, RK, KT and SY participated in the patient's care and analyzed the laboratory data; all authors have read and approved the final version of the manuscript.

Conflict of interest: the authors declare no potential conflict of interest.

Received for publication: 16 February 2015.

Revision received: 21 April 2015.

Accepted for publication: 25 May 2015.

This work is licensed under a Creative Commons Attribution NonCommercial 3.0 License (CC BYNC 3.0).

(C) Copyright K. Yamamoto et al., 2015

Licensee PAGEPress, Italy

Pediatric Reports 2015; 7:5873

doi:10.4081/pr.2015.5873

detected by throat swab. EBV-associated antibodies were not elevated. Anti J0-1 antibody and anti-nucleus antibody were negative. Fatty acid analysis using tandem mass spectrometry did not show any abnormal findings. Within 4 days, muscle weakness disappeared and her leg movement normalized (Figure 1). Resolution of her symptoms was accompanied by a CK reduction to $294 \mathrm{IU} / \mathrm{L}$. These findings demonstrate that the patient was affected with acute myositis following simultaneous infection with rotavirus and norovirus. 0 n day 8 , she was able to walk home.

\section{Discussion}

The diagnosis of benign acute myositis in children is usually made based on the typical clinical symptoms, such as symmetrical calf muscle pain, tenderness, inability or refusal to walk a few days after infectious illness, CK elevation that normalizes within 1 week. ${ }^{7}$ Although most reported cases with virus associated benign myositis were diagnosed with- 
out electrophysiological or cerebrospinal fluid examination, $2,3,7$ these studies would help to exclude other diseases such as Guillain-Barré syndrome. However, our patient demonstrated elevation of CK up to $5547 \mathrm{U} / \mathrm{L}$, which is not usually observed in Guillain-Barré syndrome. Moreover, rapid resolution of the clinical symptom associated with concomitant decline in CK level indicates that the damaged tissue was muscle, making it highly unlikely that the girl was affected with Guillain-Barré syndrome. These clinical and laboratory findings were entirely consistent with benign acute myositis as reported in the previous literature, $2,3-7$ which led to the diagnosis of our patient as acute myositis.

Acute myositis is often caused by infectious pathogens such as mycoplasma pneumonia, influenza virus and adenovirus. ${ }^{5}$ Although concurrent infection of norovirus and rotavirus in acute myositis has never been described, our patient developed myositis following infection with the combination of these viruses. Previous reports demonstrating an association between rotavirus infection and myositis on rare occasions suggest that myositis was more likely attributed to rotavirus rather than norovirus in our case..$^{2-4}$ However, the involvement of norovirus could not be excluded (see below). The mechanism responsible for rotavirus associated myositis is yet to be determined, but it is considered a consequence of direct virus invasion into muscle or an immunological reaction..$^{5,8} \mathrm{~A}$ particular subtype of rotavirus may be associated with muscle tropism, although virus subtypes were not determined in our case or in other reports. Our patient developed myositis following cessation of gastroenteritis symptoms, which is similar to other reported cases (Table 1), ${ }^{2-4,9-12}$ suggesting that an indirect mechanism is likely responsible, rather than direct invasion of the virus into skeletal muscle. The pathogenesis of acute myositis remains unknown, although the immune mediated mechanisms initiated by the hosts are suspected.2,7 Elevation of soluble IL-2R in our case suggests activation of T- cells and immune dysregulation, which might have contributed to the development of myositis. With respect to the acute myositis and the immune dysregulation, infection with parvovirus B19 is associated with autoimmune acute dermatomyositis. ${ }^{13}$ However, our patient did not demonstrate elevation auto-antibodies that are known to be involved in immune myositis. Although an association between norovirus and acute myositis has never been reported, norovirus could have been the causative pathogen for the muscle damage in our case. While the cellular tropism of noroviruses has not been defined, it has been reported that norovirus RNA was detected in the serum of infected children and in the cerebrospinal fluid in cases presenting with encephalopathy, indicating extraintestinal spread in the infected individuals. ${ }^{8}$ However, it remains to be determined whether norovirus can directly invade muscle or indirectly cause myositis. Alternatively, norovirus may have

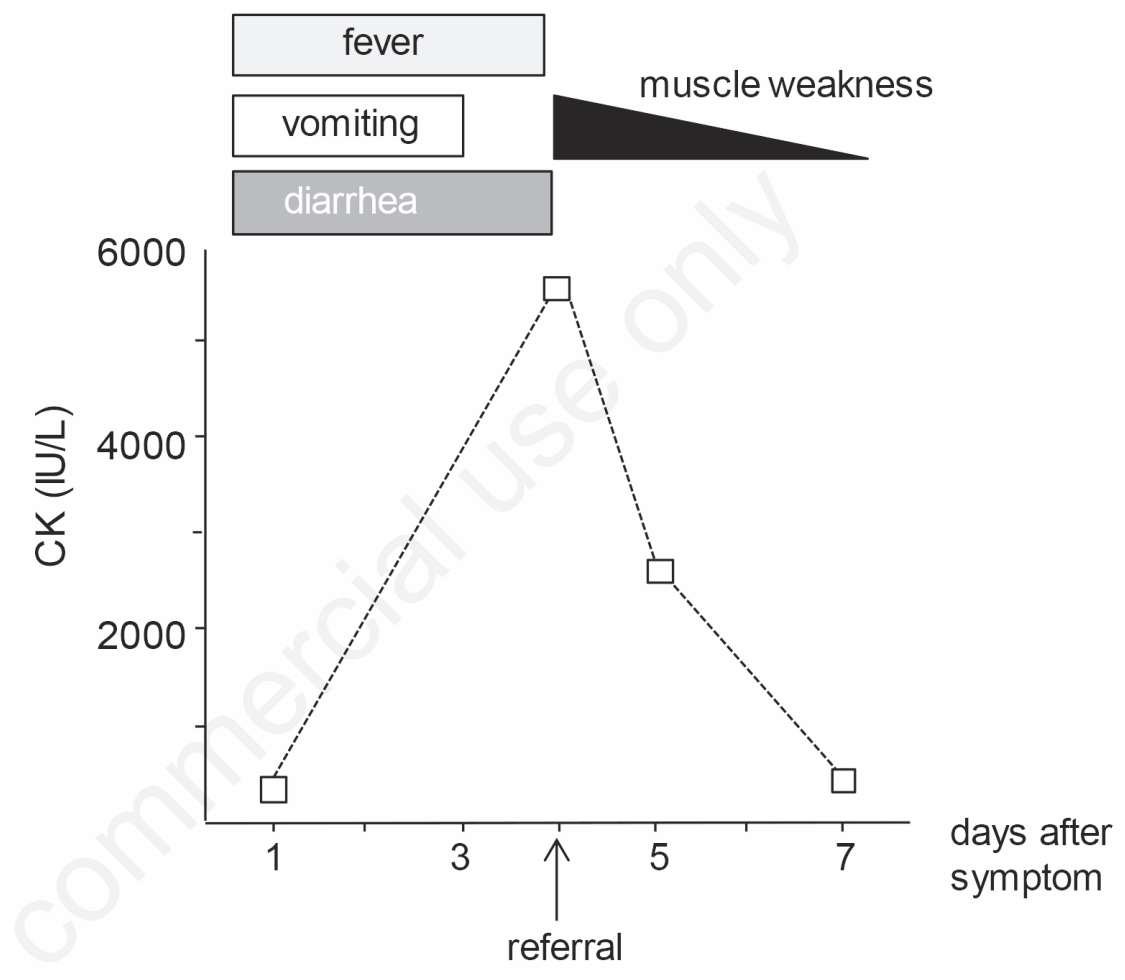

Figure 1. Clinical course and serum creatine phosphokinase activity of the patient. The patient was referred to our department on day 4 following 3 days of infusion for gastroenteritis. While she did not have fever, vomiting and/or diarrhea on the day of referral, she was not able to stand or walk. However, she recovered within 4 days without any medication.

Table 1. Cases manifesting creatine phosphokinase (CK) elevation during the course of rotavirus infection.

\begin{tabular}{|c|c|c|c|c|c|c|}
\hline Age & end & $\max$ (IU) & Symptoms & Onset day & Days to recovery & Reference \\
\hline $2.7 \mathrm{y}$ & $\mathrm{F}$ & 3675 & Unable to walk & 5 & 4 & Hattori et al. ${ }^{3}$ \\
\hline $1.2 \mathrm{y}$ & $\mathrm{F}$ & 5398 & Unable to walk & 5 & 7 & Nakazawa et al. ${ }^{9}$ \\
\hline $2 y$ & M & 7381 & Unable to walk & 6 & 13 & Bonno et al. ${ }^{2}$ \\
\hline $1 \mathrm{y}$ & M & 7952 & Cerebral palsy, macro CK, hyper CK was associated with hyper tonus. & 5 & 30 & Hasui et al. 10 \\
\hline $8 y$ & $\mathrm{~F}$ & 9861 & Inability to hold the body, encephalopathy & 3 & 5 & Fukumura et al.11 \\
\hline $7 y$ & $\mathrm{~F}$ & 5118 & Muscle weakness & 2 & 9 & Fukumura et al.11 \\
\hline $6 \mathrm{~m}$ & M & 105,959 & Encephalopathy, rhabdomyolysis & 3 & 44 & Minami et al. ${ }^{4}$ \\
\hline $1 y$ & M & 2022 & Cardiac effusion, macro CK & 3 & 15 & Oda et al. 12 \\
\hline $2.5 \mathrm{y}$ & $\mathrm{F}$ & 5547 & Unable to walk & 4 & 4 & Present case \\
\hline
\end{tabular}


modulated the immunological reaction of the patient in response to rotavirus, which subsequently resulted in myositis. Indeed, norovirus infection regulates the interferon response in mice, suggesting that noroviruses can modulate host immunological responses. 14,15

The majority of patients with rotavirus-associated myositis, as well as our patient, are over 2 years old (Table 1). In contrast, most children with rotavirus gastroenteritis are generally younger than 2 years of age, suggesting that rotavirus-induced myositis could be age dependent. However, the number of patients may be underestimated because the majority of subjects affected with rotavirus are generally infants who have yet to walk, making any gait disturbance difficult to notice. In contrast, influenza virus or mycoplasma pneumonia normally affects older children; therefore, their gait disturbance is more likely to be recognized. Blood CK activity in our case was normal before the manifestation of muscle weakness, which quickly returned to normal, thus excluding congenital muscular dystrophy or myopathy. Most cases reported so far, including ours, recovered in a short time. Yet, in rare situations, cardiac muscle damage or rhabdomyolysis is a serious complication.5,16 Although CK-MB activity was over the normal range in our case, her cardiac function was normal, suggesting that the elevation of CKMB activity in our case was most likely derived from skeletal muscle.

\section{Conclusions}

We documented a female patient with acute myositis associated with gastroenteritis induced by concomitant infection with rotavirus and norovirus. Development of myositis following cessation of gastroenteritis symptoms suggested an indirect mechanism was responsible rather than direct virus invasion. The causative pathogen was likely rotavirus in our case. However, it is possible that norovirus was also involved. In this regard, additional case accumulation is warranted to determine if norovirus can indeed result in acute myositis or if a combination of rotavirus and norovirus increases the risk of developing muscle inflammation. Because virus induced acute myositis can result in rhabdomyolysis or cardiac muscle damage, physicians should be aware of acute myositis as a potential complication of gastroenteritis induced by rotavirus or possibly norovirus.

\section{References}

1. Fukuda S, Kishi K, Yasuda K, et al. Rotavirus-associated encephalopathy with a reversible splenial lesion. Pediatr Neurol 2009;40:131-3.

2. Bonno M, Higashigawa M, Nakano T, et al. Acute myositis with transient decrease of albumin, immunoglobulin, and complement following rotavirus gastroenteritis. Acta Paediatr Jap 1998;40:82-4.

3. Hattori H, Torii S, Nagafuji H, et al. Benign acute myositis associated with rotavirus gastroenteritis. J Pediatr 1992;121:748-9.

4. Minami K, Tamura A, Komori Y, et al. Acute encephalopathy and rhabdomyolysis following rotavirus gastroenteritis. J Paediatr Child Health 2007;43:90-91.

5. Singh U, Scheld WM. Infectious etiologies of rhabdomyolysis: three case reports and review. Clin Infect Dis 1996;22:642-9.

6. Grech V, Calvagna V, Falzon A, Mifsud A. Fatal, Rotavirus-associated myocarditis and pneumonitis in a 2-year-old boy. Ann Trop Paediatr 2001;21:147-8.

7. Zafeiriou DI, Katzos G, Gombakis N, et al. Clinical features, laboratory findings and differntial diagnosis of benign acute childhood myositis. Acta Paediatr 2000; 89:1493-4

8. Warren JD, Blumbergs PC, Thompson PD. Rhabdomyolysis: a review. Muscle Nerve 2002;25:332-47.

9. Nakazawa $\mathrm{T}$, Inoue $\mathrm{S}$, Takahashi $\mathrm{H}$, Kaneko K. A case with myositis associated with rotavirus gastroenteritis. Shounika Rinsho (Japanese) 1996;49:1073-6.

10. Hasui M, Tsuiji H, Hanni et al. A cerebral palsy patient with type 2 macro creatine kinase in the serum. Shounika (Japanese) 1998;39:1065-8.

11. Fukumura $S$, Oyanagi R, Yanai $S$ et al. Two cases with myositis associated with rotavirus induced gastroenteritis. Rinsho Shouni Igaku (Japanese) 2003;51:75-7.

12. Oda K, Omura, Goto M, Seto S. A rotavirus gastroenteritis with elevated $\mathrm{CK}$ and cardiac effusion. Jpn Soc Pediatr Infect Dis Proc 2009;41:165.

13. Crowson AN, Magro CM, Dawood MR. A causal role for parvovirus B19 infection in adult dermatomyositis and other autoimmune syndromes. J Cutan Pathol 2000;27: 505-15

14. Karst SM, Zhu S, Goodfellow IG. The molecular pathology of noroviruses. J Pathol 2015;235:206-16.

15. Sánchez-Fauquier A, González-Galán V, Arroyo S, et al. Norovirus-associated encephalitis in a previously healthy 2 yearold girl. Pediatr Infect Dis J 2015;34:222-3

16. Cioc A, Nuovo GJ. In situ hybridization analysis of the myocardium for viral nucleic acids in cases of sudden, unexpected death. Modern Pathol 2002;15:5A. 